\title{
PULMONARY ASPERGILLOSIS IN IMPORTED GREAT BLUE TURACO, CORYTHAEOLA CRISTATA.
}

Omar A.S. Tamam

Department of Natural Resources, Environmental Studies and Research Institute, University of Sadat City, Sadat City Campus, Egypt.

E-mail: otamam2002@ hotmail.com

\section{ABSTRACT}

Received at: 20/12/2014

Accepted: 31/12/2014
The Great Blue Turaco (Corythaeola cristata) has been raised in captivity for over a hundred years, but their susceptibility to infectious disease is very poorly documented. The macroscopic, histopathologic, and ultrastructural changes in the respiratory tract of nine Great Blue Turacos (Corythaeola cristata) who died in captivity were investigated. Macroscopically diffuse granulomatous lesions were observed in the lung and the associated air sacs, with a grey-greenish mold on their inner surface. Histopathologic examination revealed widespread necrosis in the lungs associated with vasculitis. Microthrombi were observed in the blood vessels suggesting disseminating intravascular coagulation. Intra-alveolar hemorrhage and infiltration with macrophages, plasma cells, epithelioid cells, and multinucleate giant cells were observed. PAS staining showed hyphae and conidia, While, Ziehl-Neelsen stain was negative. Transmission electron microcopy examination revealed the presence of Aspergillus fumigatus conidia invading the pulmonary tissue as semilunar spores within the alveolar macrophages. Lysosomal degranulation was also observed in association with fragmented spores suggesting active phagocyosis. Awareness of this condition might improve the rapid and appropriate treatment of exotic birds kept in captivity.

Keywords: Aspergillosis; Lungs; Histopathology; Greater blue turaco.

\section{INTRODUCTION}

Pulmonary aspergillosis results from a range of factors including bird capture, transportation (for instance from tropical to temperate climates), overcrowding, high humidity, bad sanitation, inadequate hygiene measures, and contact with other bird species. Bozza et al. (2002) reported that Aspergilli are respiratory pathogens and pulmonary infections usually acquired through the inhalation of conidia and able to reach small airways and the alveolar space where the impaired host defense mechanisms allow hyphal germination and subsequent tissue invasion. The invasive pulmonary aspergillosis is the most common manifestation of Aspergillus fumigatus infection in immunocompromised patients and is characterized by hyphal invasion and destruction of pulmonary tissue. Resident alveolar macrophages ingest and kill resting conidia, mainly through nonoxidative mechanisms, while neutrophils use oxygen-dependent mechanisms to attack hyphae germinating from conidia that escape macrophage surveillance (Bozza et al., 2002). Murine pulmonary denderitic cells internalize conidia and hyphae of A. fumigatus both in vitro, and during infection, in vivo. Internalization occurs through distinct phagocytic mechanisms and recognition receptors. Phagocytosis of conidia induces IL-12 production, while that of hyphae induces IL-4 and IL10. In vivo, pulmonary DC, after phagocytosis of conidia or hyphae, migrate to the draining lymph nodes and spleens, undergo functional maturation, and induce selective Th priming of $\mathrm{CD} 4{ }_{-} \mathrm{T}$ lymphocytes (Bozza et al., 2002).

The Great Blue Turaco (Corythaeola cristata) is the largest turaco, and although traditionally thought to belong to the largest species in the diverse Cuculiformes order (which includes the cuckoos), DNA hybridization studies indicate that the family is better regarded as having ordinal status (Musophagiformes) (Sibley and Ahlquist, 1990). Although C. cristata is on the IUCN Red List of Endangered Species, the species is currently evaluated as being of least concern (LC) and population levels are stable. 
However, while $C$. cristata have been raised in captivity for over a hundred years, there is relatively little scientific literature on these captive exotic birds, and therefore aviculturists rely on anecdotal evidence to make management decisions. Most deaths in captivity are due to traumatic injuries or conspecific aggression, but Great Blue Turacos are also susceptible to other diseases common in captive birds (Brannion, 1993). Juvenile birds reared on calciumpoor diets can develop metabolic bone disease, and iron-storage disease has occasionally been reported (Fowler and Miller, 2007). With respect to infectious disease, the family is particularly susceptible to avian mycobacteriosis as a frequent cause of mortality (Brannion, 1993). Infection with mycobacteria in any species can be confused with Yersinia pseudotuberculosis, which is far less contagious and lethal, highlighting the need for accurate pathological and microbiological diagnosis (Fowler and Miller, 2007).

The objective of the present study is to assess the clinical, histopathological, microbiological, and ultrastructural analysis of nine Great Blue Turacos underwent necropsy after a fatal outbreak in a group of birds kept in captivity in Egypt.

\section{MATERIALS and METHODS}

Nine birds out of a total of 35 birds kept in captivity in Egypt died unexpectedly over the course of two months, having been kept in close confinement over the preceding two weeks for the purpose of travel between aviaries. In order to establish the cause of death, the birds underwent a thorough post-mortem examination. Since the study did not use laboratory animals and was performed as part of the normal investigation of natural disease, no ethical approval was required. Lung tissues with macroscopic lesions suggestive of fungal infection were taken and immediately fixed in $10 \%$ buffered formalin, paraffin-wax embedded for histological examination. In addition, samples were taken from the liver, kidneys, heart, skeletal muscle, crop, pro-ventriculus, gizzard, intestine, pancreas, and spleen for histopathological examination. Fixed tissues were processed using standard histology protocols, sectioned at $3 \mu \mathrm{m}$, and stained with hematoxylin and eosin, periodic acid Schiff (PAS), and Ziehl-Neelsen (ZN) for subsequent microscopic analysis and photomicrography using a digital camera. A fresh portion of tissue from the same area as the histopathology sample area was sown in Sabouraud Dextrose Agar (SDA) and incubated at $37^{\circ} \mathrm{C}$ for 4 to 10 days for the culture and detection of fungi. Further samples were cultured in nutrient broth for one day then cultured on blood agar in order to culture and detect pathogenic bacteria.
Lung samples were perfused with modified Karnovsky solution containing $2.5 \%$ glutaraldehyde and $2 \%$ paraformaldehyde in a $2.5 \%$ sodium cacodylate buffer at a final concentration of $0.1 \mathrm{M}$ ( $\mathrm{pH} 7.3$ ), and fixed in the same solution for 12 hours at $4{ }^{\circ} \mathrm{C}$. The specimens were post-fixed in $2 \%$ osmium tetroxide solution, rinsed in distilled water, and immersed in $2 \%$ tannic acid solution for 1 hour at room temperature. The tissues were dehydrated in a graded series of ethanol and propylene oxide and embedded in Spurr resin. Thick sections were cut using a Porter Blum ultra microtome using glass blades and stained with toluidine blue solution for light microscopic analysis. An Ultra-Cut Reichert ultra-microtome with a diamond knife was used to cut thin sections (Reichert Technologies Inc., Depew, NY 14043, USA). The ultrathin sections were mounted on 200 and 300 mesh grids, and counterstained with uranyl acetate and lead citrate. TEM was performed using the JEM100S electron microscope (JEOL Ltd., Akishima, Japan) at $100 \mathrm{kV}$.

\section{RESULTS}

A: Clinical picture: Over a period of two months there were nine unexplained Great Blue Turaco deaths in a group of 35 (25.7\% mortality). Prior to death, the birds were reported to be listless, off their food, unable to fly, and exhibited anti-social behavior. They were unresponsive to treatment with amoxicillin (Pfizer $100 \mathrm{mg} / \mathrm{kg} \mathrm{BW}$ for five days) and colistin (Alexandria $30 \mathrm{mg} / \mathrm{kg}$ bw for 5 days).

B: Post-mortem examination: Different lesions showed at different sites, and at differing stages of evolution. Diffuse granulomatous lesions were observed in the air sacs. Lesions ranged in size and character. Some were small and miliary, while others were larger with necrotic foci (up to $2 \mathrm{~cm}$ in diameter). The larger lesions were white and dry, and protruded from the surface of the affected organ with a grey-green mold on the inner surface (Figure 1). In two cases, only small lesions (2-4 $\mathrm{mm}$ in diameter) were observed in the lungs. These lesions demonstrate yellowish caseous pulmonary nodules identified as fungal granulomas of approximately 0.1 $0.5 \mathrm{~cm}$ in diameter (Figure 2). Fig.3 showed several small scattered nodules on the air sacs.

C: Histopathologic findings: Granulomatous inflammatory lesions of pulmonary aspergillosis were observed. Mycobacteriosis was excluded by negative Ziehl-Neelsen staining. The lesions had central caseous necrosis containing a mass of mycelia bordered by radiating hyphae, and variable numbers of epithelioid macrophages, lymphocytes, fibroblasts, and multinucleate giant cells surrounding the caseous material (Figs.4;5). Microthrombi were observed in 
the blood vessels (Fig.6). Hemorrhagic exudates was noticed and expressed by the presence of nucleated red blood cells (Fig.7). The air sac was thickened due to layers of degenerate heterophils and aspergillus hyphae, but also clusters of amebic trophozoites that were either spherical or oval-shaped (15 - 20 micrometer in diameter). They had a thin cell membrane and single nucleus with prominent nuclear borders and a central karyosome; binary fission was noted in some of the trophozoites (Figure 8), typical of Entamoeba histolytica. These organisms were apparent in all nine birds, and systemic protozoal infection was ruled out by microscopic examination of all the organs, which did not show any abnormalities. PAS-stained tissues, the fungal hyphae were clearly septate, with or without accompanying conidial heads on the surface of air-sacs and pleura (Fig.9).

\section{D: Transmission electron microscopic} examination: The lung tissue revealed the presence circumscribed colonies surrounded with membrane in the alveoli (Fig.10). Large numbers of macrophages containing numerous lysosomes scattered throughout the cytoplasm and surround semilunar engulfed spores (Fig.11). Fragmented spores in the alveolar macrophages and several degranulated lysosomes were observed. Cytoplasmic processes, or pseudopods, were seen at the cell surface, some of which could be seen ingesting large numbers of fungal conidia into the plasma membrane-derived phagosomes, which fuse with lysosomes to form phagolysosomes (Fig.12). No protazoa were seen by TEM.

E. Microbiological analysis: Detected Aspergillus fumigatus in these lesions and excluded the concurrent presence of other bacterial pathogens. Identification of A. fumigatus was based predominantly upon the morphology of the conidia and conidiophores, with chains of green echinulate conidia produced from greenish hyphae. The organisms grew quickly on SDA with colonies reaching $1-2 \mathrm{~cm}$ after 10 days.

\section{DISCUSSION}

To our knowledge there are no formal descriptions of aspergillosis occurring in the Great Blue Turaco, Corythaeola cristata. The present study describes Aspergillus fumigatus infection and its histopathologic and ultrastructural features in several imported Great Blue Turacos who died in captivity. In addition, some of these lesions were associated with concurrent infection with the parasitic infection Entamoeba histolytica. Amebiasis can be caused by parasitic infection with Entamoeba histolytica, among other pathogenic amoebae. The organism's definitive host is the human and this particular species has not previously been described in exotic birds, although another Entamoeba species has been idenntified in ostriches (E. struthionis) (Martinez-Diaz et al., 2000; Ponce Gordo et al., 2004).

Aspergillosis is a fungal disease caused by fungi of the genus Aspergillus, in particular A. fumigatus and A. flavescens. It has been described in many farm and wild bird species, both in the wild and in captivity (Guillot and Chermette, 2001; Tell, 2005). A. fumigatus accounts for over $95 \%$ of cases in avian infections (Tell, 2005). The target organs of the organism are the lungs and the air sacs, but systemic aspergillosis can also affect the liver, kidney, encephalon, bones, skin and eyes (Raja and Singh, 2006; Trullas et al., 2005). Histopathologically, the lesions are characterized by two different patterns: a granulomatous form with internally-septate hyphae, that typically develop into non-aerated parenchyma, and a non-encapsulated diffuse form, containing sporangia with a typical morphology, located in the lung or, even more frequently, in the air sacs (Nardoni et al., 2006).

In the present study, granulomas consisted of caseous necrosis surrounded by epithelioid cells, lymphocytes, fibroblasts, and multinucleated giant cells. There was no evidence for mycobacterium as indicated by negative staining reaction of Zheil Neelson stain. Birds are very susceptible to Aspergillus infection (Lair-Fulleringer et al., 2003), particularly in response to stressors such as starvation, thermal and adverse environmental conditions, or trauma-related immunosuppression, which allows colonization of tissues by opportunistic fungi (Akan et al., 2002; Carrasco et al., 2001). These birds had not been given prophylactic antifungals while being transported to Egypt, and although drugs were administered when symptoms were noted these were not effective.

Histopathology remains a useful tool for the diagnosis of aspergillosis in birds, since antigen and antibody tests, although variable, are known to have low sensitivity and specificity (Chan et al., 2002; Hope et al., 2005). The focal, multifocal, and disseminated forms of the disease patterns seen in this case series are known to occur, and the pattern of massive granulomas with necrotic cores is a typical histopathologic pattern.

In the present study, TEM revealed the presence of conidia in the alveolar tissues as well as inside the alveolar macrophages as semilunar shaped. Lysosomes and pseudopodia were evident feature of the alveolar macrophages. Moreover, lysosomal degranulation in association with the fragmented spores and suggested active phagocytosis had occurred. Merkow et al. (1971) stated that the 
cytoplasm of alveolar macrophages that had not phagocytized spores contained numerous widely dispersed lysosomes (granules) with variable staining affinity and inclusions. While, macrophages phagocytose spores or hyphae had in an apparent "degranulation" of those portions of the cytoplasm distal to the spores.

The factors that contribute to the susceptibility of birds to Aspergillus spp. are also likely to have contributed to the co-infection with E. histolytica. Anatomically, birds lack an epiglottis and therefore particulate matter has easy access to the lower respiratory tract, they lack a diaphragm and therefore cannot expel foreign debris by coughing, and pseudostratified epithelium has only limited distribution in the respiratory tract within the air sacs; therefore there is no effective mucociliary escaltor to remove debris from the upper airways. The characteristic endosomal-lysosomal phagocytic response seen in this study by TEM is in contrast to the myeloperoxidase and oxidative mechanisms seen in mammals and is less effective for killing fungal hyphae (Harmon, 1998). This inferior innate immune mechanism may explain the presence of aspergillosis seen in this case series, and might also explain secondary infection and susceptibility to $E$. histolytica. However, whether E. histolytica represents superadded infection due to primary aspergillosis, or is pathogenic in its own right, remains to be determined. However, we speculate that amebiasis represents a secondary infection contributing to the severity of the disease in these birds and the lethality.

\section{CONCLUSIONS}

In conclusion, although aspergillosis has been described in other bird species, this is the first reported case of mycotic pneumonia caused by A. fumigatus in the Great Blue Turaco. Further studies are required to show the sensitivity of this species against the mycotic infection and to search for the suitable treatments. These findings highlight the importance in accurate and systematic pathological diagnosis of avian disease and provide new information to help manage disease outbreaks in exotic birds kept in captivity.

\section{FIGURE LEGENDS}

Fig. 1: Growth appearance of the granuloma showed thickened grayish green mycotic material (arrow) surrounded by thick wall.

Fig. 2: Growth appearance in the lungs demonstrate yellowish and caseous pulmonary nodules identified as fungal granulomas of approximately $0.1-0.5 \mathrm{~cm}$ in diameter (arrow).

Fig.3: Growth appearance showing several small scattered nodules on the air sacs (arrow).

Fig.4: Light micrograph of Granulomatous lesions showing presence of variable numbers of epithelioid cells, lymphocytes, fibroblasts, and multinucleated giant cells (arrows). H\&E X100.

Fig.5: Light micrograph of Lungs showing radiating hyphae of Aspergillus fumigatus (long arrow) as well as heavy epitheliod cellular reaction (head arrow). $\mathrm{H} \& \mathrm{E} X 100$.

Fig. 6: Light micrograph of Blood vessels of the lungs showed presence of small microthromi (long arrow) as well as epitheliod cells (head arrow). H\&E $\mathrm{X} 100$.

Fig.7: Light micrograph of lungs showed alvealor hemorrhage expressed by the presence of nucleated red blood cells (star). H\&E X400.

Fig. 8: Light micrograph of Lungs showed caseaous necrotic center (star) and few amebic trophozoites appeared spherical or oval-shaped (15 - 20 micrometer in diameter) with a thin cell membrane (arrow) (H\&E X400).

Fig. 9: Light micrograph of short slender branching septated red hyphae (long arrow) with radiating colonies on the alveoli (head arrow). PAS stain. X400

Fig. 10: TEM showed collection of circumscribed colonies surrounded with membrane in the alveoli. Uranyl acetate and Lead citrate. 2000x.

Fig. 11: TEM showed well-demarcated semilunar spores (star) within the alveaolar macrophage, several lysosomes were also observed (head arrow). Uranyl acetate and Lead citrate. 2000x.

Fig. 12: TEM showed alveolar macrophages had fragmented spores (star) surrounded with empty lysosomes (head arrow), pseudopodia (long arrow). Uranyl acetate and Lead citrate. 2000x. 


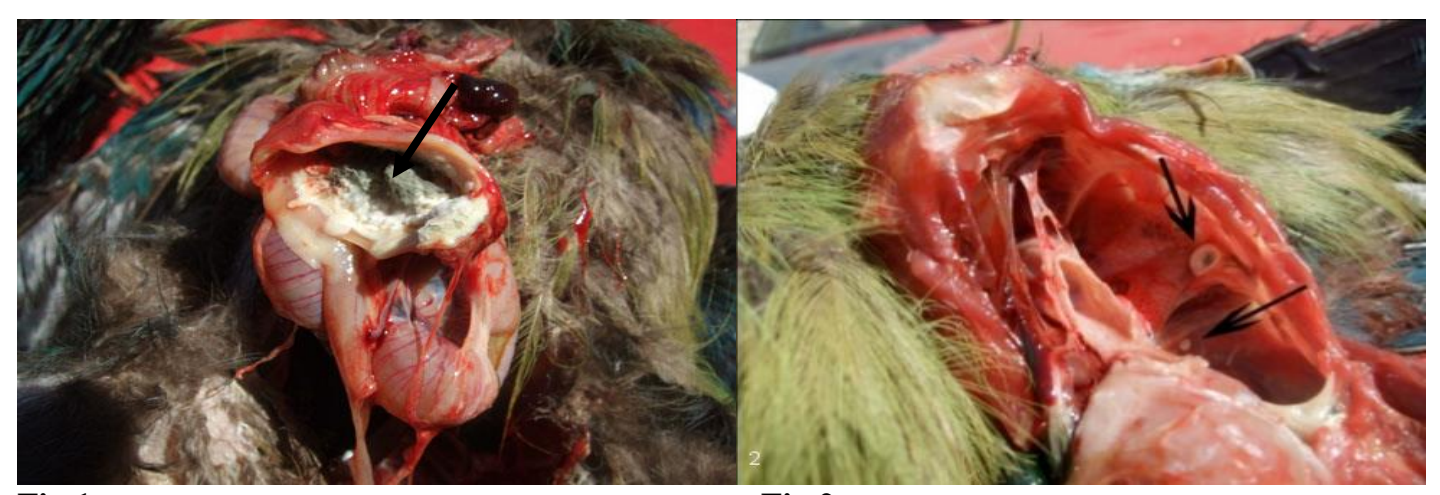

Fig.1

Fig.2

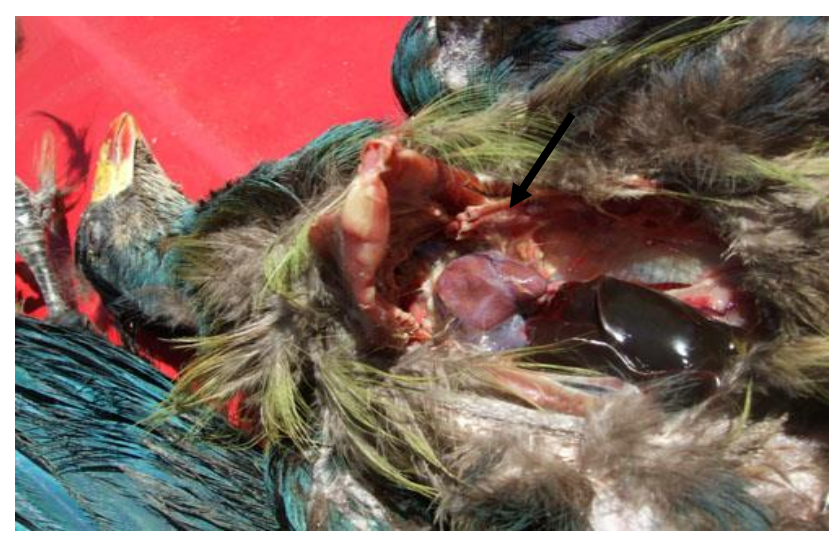

Fig.3

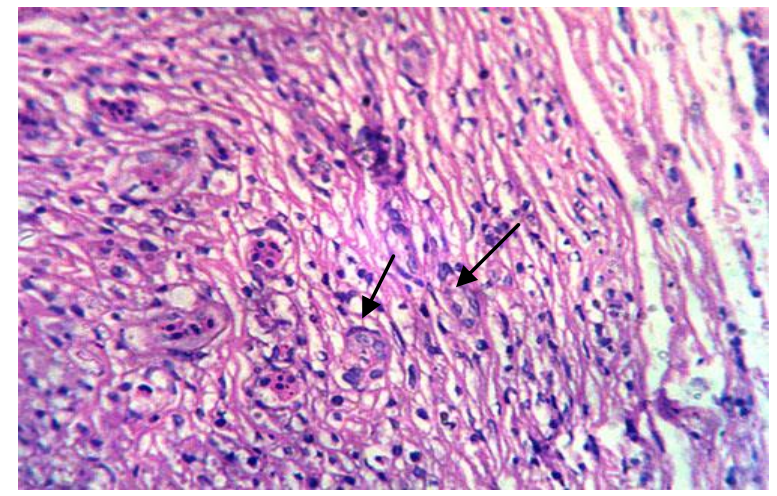

Fig. 4

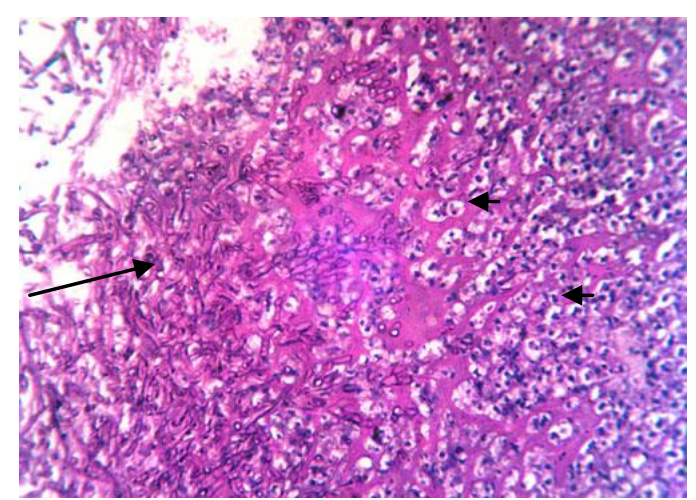

Fig.5

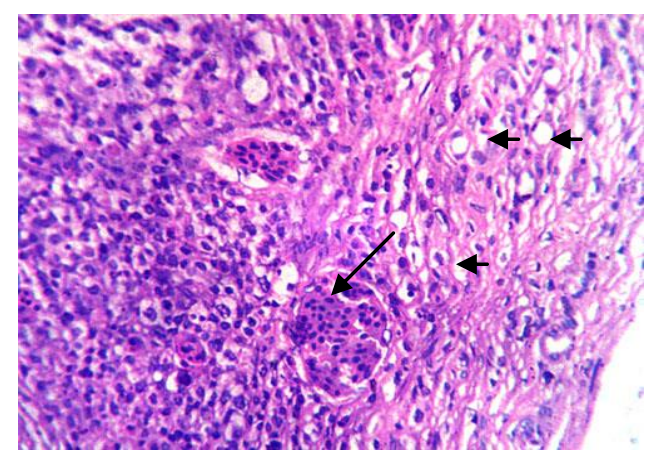

Fig.6

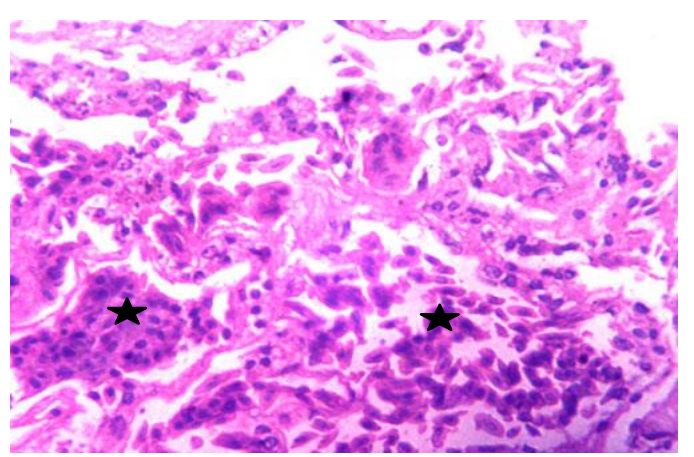

Fig.7 
$\underline{\text { Assiut Vet. Med. J. Vol. } 61 \text { No. } 144 \text { January } 2015}$

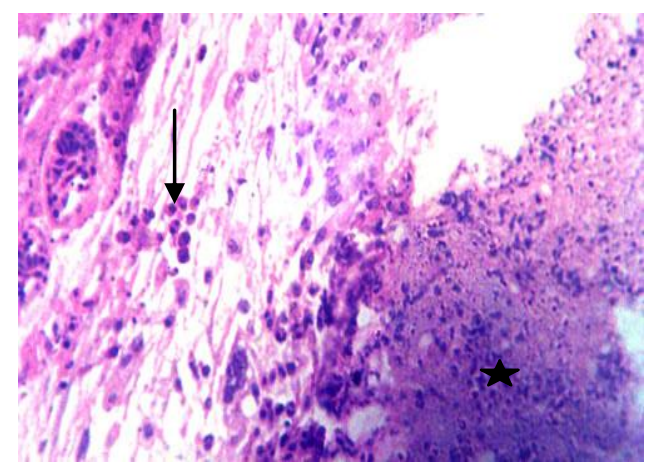

Fig.8

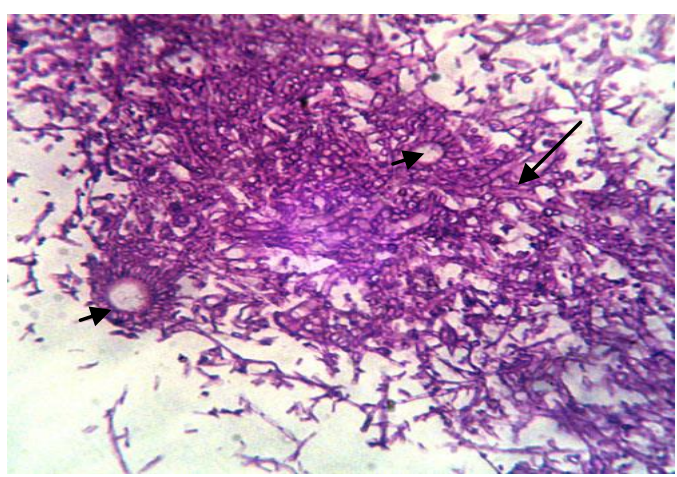

Fig.9
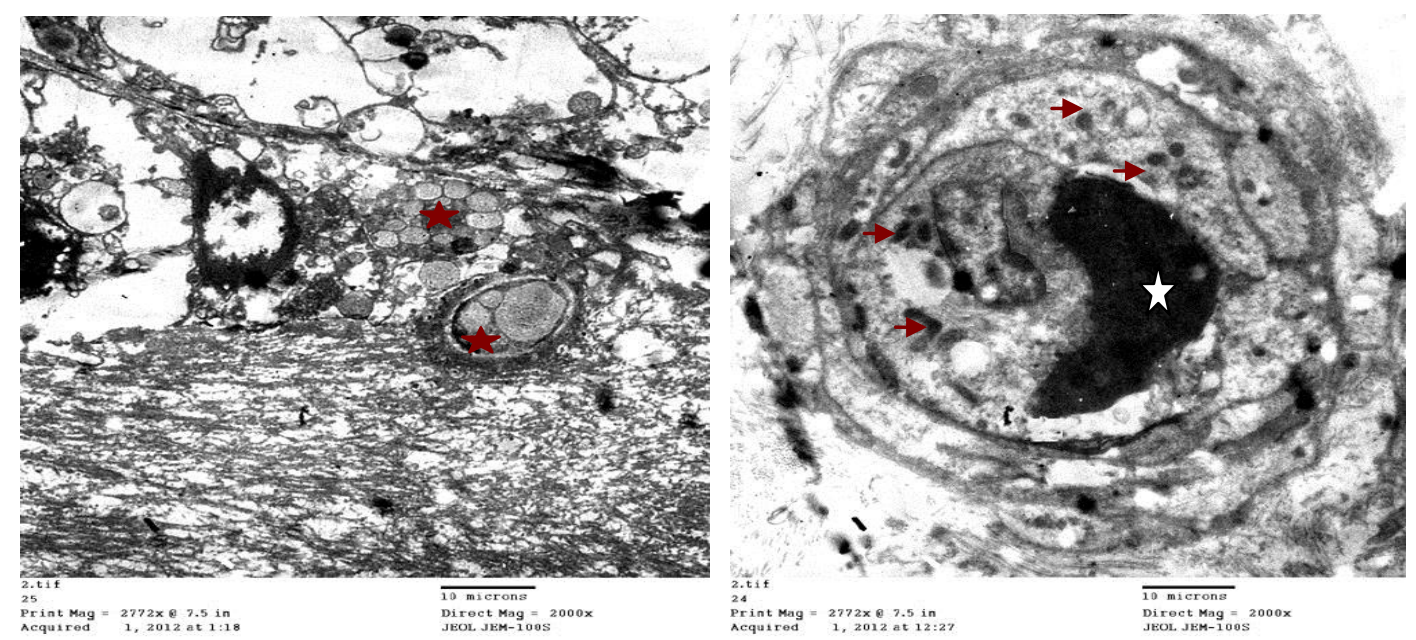

Fig. 10

Fig.11

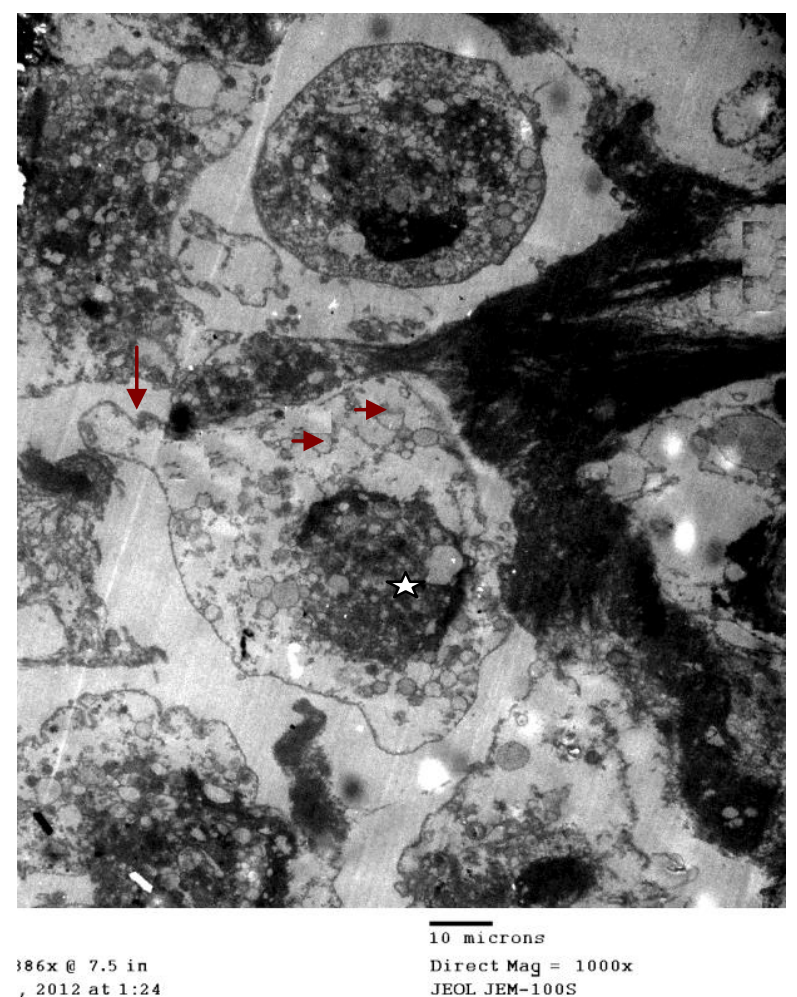

Fig.12 


\section{REFERENCES}

Akan, M.; Haziroglu, R.; Ilhan, Z.; Sareyyupoglu, B. and Tunca, $R$. (2002): A case of aspergillosis in a broiler breeder flock. Avian Dis, 46: 497501.

Atasever, A. and Gumussoy, KS. (2004): Pathological, clinical and mycological findings in experimental aspergillosis infections of starlings. J Vet. Med. A Physiol Pathol Clin. Med., 51: 19-22.

Bozza, S.; Roberta Gaziano; Antonio Spreca; Angela Bacci; Claudia Montagnoli; Paolo di Francesco, and Luigina Romani (2002): Dendritic Cells Transport Conidia and Hyphae of Aspergillus fumigatus from the Airways to the Draining Lymph Nodes and Initiate Disparate Th Responses to the Fungus. J Immunol 168: 1362-1371.

Brannion, R. (1993): Diseases of turacos, go-away birds, and plantain-eaters, Toronto, W.B. Saunders.

Carrasco, L.; Lima, JS.Jr.; Halfen, DC.; Salguero, FJ.; Sanchez-Cordon, $P$. and Becker, G. (2001): Systemic aspergillosis in an oiled magallanic penguin (Spheniscus magellanicus). J. Vet. Med. B. Infect. Dis. Vet. Public Health, 48: 551-554.

Chan, CM, Woo, PC.; Leung, AS.; Lau, SK.; Che, $X Y$.; Cao, L. and Yuen, KY. (2002): Detection of antibodies specific to an antigenic cell wall galactomannoprotein for serodiagnosis of Aspergillus fumigatus aspergillosis. J Clin Microbiol, 40: 2041-2045.

Fowler, ME. and Miller, RE. (2007): Zoo and Wild Animal Medicine Current Therapy, Elsevier Health Sciences.

Guillot, J. and Chermette, R. (2001): [Aspergillosis in birds]. Rev Prat, 51: 704-707.

Hara, A.; Hirose, Y.; Mori, H.; Iwao, H.; Kato, T. and Kusuhara, Y. (2004): Cytopathologic and genetic diagnosis of pulmonary amebiasis: a case report. Acta Cytol, 48: 547-550.

Harmon, BG. (1998): Avian heterophils in inflammation and disease resistance. Poult Sci, 77: 972-977.

Hope, WW.; Walsh, TJ. and Denning, DW. (2005): Laboratory diagnosis of invasive aspergillosis. Lancet Infect Dis, 5: 609-622.
Lair-Fulleringer, S.; Guillot, J.; Desterke, C.; Seguin, D.; Warin, S.; Bezille, A.; Chermette, R. and Bretagne, S. (2003): Differentiation between isolates of Aspergillus fumigatus from breeding turkeys and their environment by genotyping with microsatellite markers. J. Clin. Microbiol, 41: 1798-1800.

Martínez-Díaz, R.A.; Herrera, S.; Castro, A. and Ponce, $\quad$ F. (2000): $\quad$ Entamoeba sp. (Sarcomastigophora: Endamoebidae) from ostriches (Struthio camelus) (Aves: Struthionidae). Vet. Parasitol 92, 173-179.

Merkow, L.M.; Sheldon, M.; Epstein, Herschel Sidransky; Ethel Verney, and Matias Pardo (1971): The Pathogenesis of Experimental Pulmonary Aspergillosis. An Ultrastructural Study of Alveolar Macraphages after Phagocytosis of A Flavus Spores In Vivo. American Journal of Pathology 62, No. 1, 5868.

Nardoni, S.; Ceccherelli, R.; Rossi, G. and Mancianti, F. (2006): Aspergillosis in Larus cachinnans micaellis: survey of eight cases. Mycopathologia, 161: 317-321.

Ponce-Gordo, F.; Martínez-Díaz, R.A. and Herrera, S. (2004): Entamoeba struthionis n. sp. (Sarcomastigophora: Endamoebidae) from ostriches (Struthio camelus). Vet Parasitol 119, 327-335.

Raja, NS. and Singh, NN. (2006): Disseminated invasive aspergillosis in an apparently immunocompetent host. J. Microbiol Immunol Infect, 39: 73-77.

Sibley, CG. and Ahlquist, JE. (1990): The Phylogeny and Classification of Birds: A Study in Molecular Evolution, Yale University Press.

Tell, LA. (2005): Aspergillosis in mammals and birds: impact on veterinary medicine. Med Mycol, 43 Suppl 1: S71-73.

Trullas, JC.; Cervera, C.; Benito, N.; De La Bellacasa, JP.; Agusti, C.; Rovira, M.; Mas, A.; Navasa, M.; Cofan, F.; Ricart, MJ.; PerezVilla, F. and Moreno, A. (2005): Invasive pulmonary aspergillosis in solid organ and bone marrow transplant recipients. Transplant Proc, 37: 4091-4093.

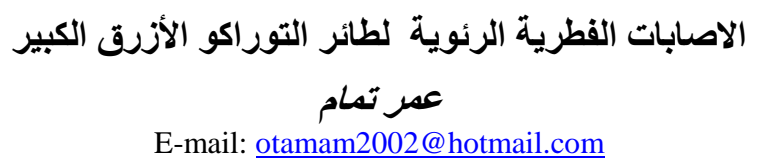

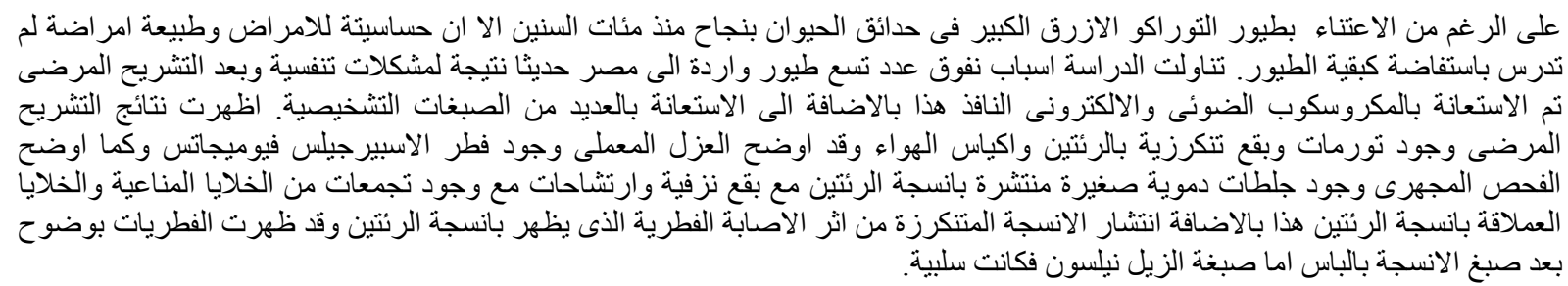

